\title{
Reactivity of dog sera to whole-cell or recombinant antigens of Borrelia burgdorferi by ELISA and immunoblot analysis
}

\author{
LOUIS A. MAGNARELLI, STEVEN A. LEVY*, JACOB W. IJDO†, CAIYUN WU†, \\ STEVEN J. PADULA $\ddagger$ and EROL FIKRIG†
}

Department of Entomology, Connecticut Agricultural Experiment Station, New Haven, CT 06504, *Durham Veterinary Hospital, 178 Parmelee Hill Road, Durham, CT, †Section of Rheumatology, Department of Internal Medicine, Yale University School of Medicine, New Haven, CT 06520 and \$Division of Rheumatic Diseases, Department of Medicine, University of Connecticut Health Center, Farmington, CT 06030, USA

\begin{abstract}
Enzyme-linked immunosorbent assays (ELISAs) with separate preparations of 10 purified recombinant antigens of Borrelia burgdorferi sensu stricto were used to test sera from 36 dogs not vaccinated with whole cells of this agent and from five dogs vaccinated with whole-cell $B$. burgdorferi bacteria. All dogs lived in tick-infested areas of Connecticut and south-eastern New York state, USA. The non-vaccinated dogs had limb or joint disorder, lameness and fever during the period 1984-1991 and had antibodies to $B$. burgdorferi, as determined by a polyvalent ELISA with whole-cell antigen. In reanalyses of sera for total immunoglobulins in ELISAs with recombinant antigens, reactions were most frequently recorded when outer-surface protein (Osp) F, protein (p)35, p37, p39 and p-41G (a flagellin component) were tested separately. Western immunoblots of a subset of 16 sera, positive by ELISA with whole-cell antigen and representing a range of antibody titres (640-40 960), verified immune responses to these or other lysed whole-cell antigens. Sera from vaccinated dogs contained antibodies to OspA, OspB, p22, p37 and p41-G. Therefore, serological reactions to OspF, p35 and p39 were the most important indicators of natural exposure to $B$. burgdorferi. Serum reactivities to these recombinant antigens in ELISAs can be used to help identify possible natural infections of canine borreliosis in dogs not vaccinated with whole-cell $B$. burgdorferi and to provide information on the geographic distribution of this bacterium.
\end{abstract}

\section{Introduction}

Lyme borreliosis occurs in North America and Eurasia where Ixodes pacificus, I. persulcatus, I. ricinus and I. scapularis ticks abound. In dogs, limb and joint disorders associated with borreliosis can result in gait abnormalities and reluctance to move [1-4]. Renal, cardiac and neurological disorders occur less frequently [5-7] in canines. Other clinical signs include fever, lethargy and loss of appetite. Dogs living in tickinfested areas where human cases of Lyme borreliosis occur produce antibodies to Borrelia burgdorferi sensu lato [8-12]. In regions where Lyme borreliosis is rare or not known to occur, many dogs are not vaccinated for this disease. If dogs have not been immunised with whole-cell vaccines (ie., inactivated B. burgdorferi),

Received 29 Jan. 2001; accepted 9 April 2001.

Corresponding author: Dr L. A. Magnarelli (e-mail: louis. magnarelli@po.state.ct.us). results of serological testing can reveal whether or not B. burgdorferi occurs in certain communities. Such epidemiological information is particularly useful in identifying new sites where Lyme borreliosis has become endemic. As the geographical ranges of this zoonotic disease continue to expand in North America and Eurasia, there is a need for continued surveillance.

The use of whole-cell $B$. burgdorferi antigen in antibody tests can result in false positive reactions [13]. In earlier studies [12], the inclusion of recombinant antigens in separate enzyme-linked immunosorbent assays (ELISAs) was useful in conducting more specific serological testing of dog sera to determine if these animals had been naturally exposed to $B$. burgdorferi. Since then, new, highly specific recombinant antigens have been produced or made available for evaluation. Compared with other serological tests, the ELISA is easier to standardise and, with automation, large numbers of sera can be analysed. The main 
purpose of the study reported here was to further evaluate the use of purified recombinant antigens of $B$. burgdorferi in ELISAs to determine which antigens are diagnostically important and to compare these results with those obtained by performing immunoblotting methods for non-vaccinated, naturally exposed dogs.

\section{Materials and methods}

\section{Sources of serum samples and clinical data}

Veterinarians obtained 36 blood samples from 36 dogs that lived in tick-infested areas of Connecticut and south-eastern New York state, USA, during the period 1984-1991, as reported previously [12]. These dogs, of mixed breeds and different ages, were not vaccinated with whole-cell B. burgdorferi bacterin. All sera were obtained in a passive surveillance programme from dogs frequently bitten by ticks and diagnosed with canine borreliosis by having swollen joints, signs of pain on manipulation, lameness and fever [3, 11, 12]. Blood samples, collected before antibiotic treatment and within 4 weeks of the onset of illness, were positive for antibodies to B. burgdorferi, as determined previously by a polyvalent ELISA [12]. It is not known how many dogs had active infections at the times blood samples were collected. A further $12 \mathrm{dog}$ sera from Wyoming (USA), available from earlier work [12], were included as negative controls. There were no signs of illness in these subjects and no evidence of $I$. scapularis ticks or human cases of Lyme borreliosis in the areas of Wyoming where these dogs lived. Three positive control sera, selected from a group previously found to be positive by ELISA and Western blot analysis [14], were used throughout laboratory testing. All sera obtained from dogs not vaccinated with $B$. burgdorferi had been stored at $-60^{\circ} \mathrm{C}$ at the Connecticut Agricultural Experiment Station before re-analyses in 1998 and 1999.

Five dogs of varying breeds in Middlesex County, CT, USA were inoculated subcutaneously during 2000 with whole-cell inactivated B. burgdorferi bacterin (Fort Dodge Animal Health, Overland Park, KS, USA) as directed by the manufacturer. Blood samples were collected from each dog before vaccination when the dogs were 8-12 weeks old. A second vaccination was administered to each dog 2-4 weeks later, and blood samples were collected 4 weeks later. Serum samples were stored at $-60^{\circ} \mathrm{C}$ until analysed. There was no known dog exposure to ticks.

\section{Serological testing}

A polyvalent ELISA, developed earlier and evaluated for sensitivity and specificity [4, 12, 14], was used to detect total immunoglobulins to whole-cell (strain 2591) or recombinant antigens of B. burgdorferi sensu stricto. Results of an ELISA with whole-cell antigen compared favourably with those obtained by indirect fluorescent antibody (IFA) analyses [4]. The following recombinant antigens were tested separately with dog sera and horseradish peroxidase-labelled antibodies: OspA (31 kDa), OspB (34 kDa), OspC (23 kDa), OspE (19 kDa), OspF (29 kDa), protein (p) 22, p35 (47-kDa fibronectin-binding protein), p37, p39 and p41-G (the $13-\mathrm{kDa}$ central fragment of flagellin). Recombinant antigens were produced at Yale University (p22, p35, p37, p41-G, OspA, OspB, OspE, and OspF) or the University of Connecticut (p39, OspC) and were cloned and expressed as fusion proteins in Escherichia coli with glutathione S-transferase [12, 14-19]. Antigens for OspC and p22 had similar molecular mass but were different protein components of $B$. burgdorferi. The p39 antigen was produced from DNA of strain 2591, a rodent isolate from Connecticut, after amplification by PCR methods with primers (upstream primer 5'TAGTGGTAAAGGTACTCTT-3' and downstream primer 5'-TTAAATAAATTCTTTAAGAAAC-3') based on a previously published sequence [20] (GenBank accession no. L24194). Details of the production of whole-cell and recombinant antigens and their use along with controls in ELISAs have been described previously [12, 14-19].

Tests on specificity were conducted earlier and showed little or no cross-reactivity when sera with antibodies to Ehrlichia canis or Leptospira spp. were screened with whole-cell or recombinant antigens of B. burgdorferi by ELISA or IFA analyses [4, 10, 12, 14]. However, additional tests on specificity were performed to check for possible cross-reactivity of Ehrlichia equi antibodies with p22, p35, p37 and p39 antigens not analysed earlier [12]. A dog serum sample with antibodies to E. equi (homologous titre 5120) and lacking antibodies to whole-cell B. burgdorferi and one serum from a horse inoculated with $E$. equi (homologous titre 20480) and with no antibodies to $B$. burgdorferi were screened with recombinant antigens in separate ELISAs as before [12]. Sera with ehrlichial antibodies were tested because of possible reactivities to heat-shock proteins shared between these bacteria and B. burgdorferi. A positive rabbit serum sample for antibodies to $B$. burgdorferi (titre 10240), rabbit antisera to Leptospira interrogans serovars canicola (strain Mouton) and icterohaemorrhagiae (strain CF-1), rabbit antiserum to Treponema pallidum, two dog sera with antibodies to Rickettsia rickettsii and a dog serum with antibodies to E. canis were tested by ELISAs with separate whole-cell or recombinant antigens. Homologous antibody titres for these sera ranged from 320 to 10240. Details on the sources of these sera, testing procedures and prior results of serological analyses have been reported $[4,10-12,21,22]$.

Twelve negative control sera from Wyoming were used to calculate cut-off values for positive results in ELISAs. Critical regions for seropositive results in assays with OspA, OspB, OspC, OspE, OspF and p41$\mathrm{G}$ antigens have been reported $[12,14]$. For recombi- 
nant p22, p35, p37 and p39 antigens, a protein concentration of $3 \mu \mathrm{g} / \mathrm{ml}$ was most suitable for optimal serological reactivity of positive control sera. The negative control sera were diluted to 1 in 160,1 in 320 and $\geqslant 1$ in 640 , and net absorbance readings were recorded as described earlier [4, 12]. Statistical analyses (3 SD plus the mean) of net absorbance values of respective datasets were used to calculate critical regions. For the p22 antigen, net optical density (OD) values of $0.05,0.04$ and 0.04 were considered positive at serum dilutions of 1 in 160,1 in 320 and $\geqslant 1$ in 640 . For the p35 and p37 antigens, a net OD value of 0.04 indicated antibody presence at all serum dilutions. Cutoff values for the ELISA with the p39 antigen were higher $(0.26,0.15$ and 0.11$)$. The commercially produced horseradish peroxidase-conjugated goat antidog IgG (H\&L chains-specific) reagent (Kirkegaard \& Perry Laboratories, Gaithersburg, MD, USA) was diluted to 1 in 10000 in phosphate-buffered saline solution (PBSS) $\mathrm{pH}$ 7.2. All polystyrene plates contained positive and negative serum controls and checks for PBSS, glutathione and peroxidase-labelled antibodies.

Immunoblotting procedures, with lysed whole-cell $B$. burgdorferi antigens, were used to identify protein banding patterns and to compare results of serum reactivities in ELISAs with whole-cell or recombinant antigens. A subset of 16 sera, chosen for Western blot analysis, had reacted positively in preliminary ELISAs with whole-cell antigen and included samples with low (640) or high (40960) antibody titres. These sera were selected along with four negative control sera without knowledge of reactivities to recombinant antigens in ELISAs. The materials and procedures used to perform immunoblots have been reported [14]. Briefly, dog sera were diluted in PBSS to 1 in 100 and tested with lysed whole-cell B. burgdorferi (strain B 297) proteins, which had been resolved in acrylamide $12 \%$ gels by SDS-PAGE and transferred to nitrocellulose membranes. Strain B 297, a human isolate from Connecticut, is very closely related to Connecticut strain 2591 and other North American isolates [14, 23]. Both Connecticut isolates share several key immunodominant proteins with each other and with European strains $[24,25]$. In analyses of strains from the USA, the former Soviet Union and Japan [26], results of ELISAs for North American human sera were similar. The blocking reagent for blots was PBSS with non-fat dried milk 5\%. An alkaline phosphatase-labelled goat antiserum H \& L chains specific (Kirkegaard \& Perry Laboratories) was diluted to 1 in 2000 in PBSS containing bovine serum albumin 5\% against dog immunoglobulin, and blots were developed and subsequently quenched in distilled water. All analyses included positive and negative dog control sera and molecular mass standards (BioRad Laboratories, Hercules, CA, USA). Positive and negative human sera [26] were included as additional controls to verify reactivity of whole-cell and recombinant antigens. The positive human sera, from patients who had erythema migrans and later manifestations (arthritis) of Lyme borreliosis in Connecticut, were reactive in ELISAs with sonicated whole-cell antigens of B. burgdorferi and had been confirmed as positive by immunoblotting. A positive immunoblot for dog sera was defined as distinct antibody reactivity to two or more highly specific, immunodominant proteins. This is consistent with standards used before [13].

\section{Statistical analyses}

Statistically significant differences in proportions of positive sera were determined by performing a z-test (SigmaStat, SPSS, Chicago, IL, USA). The Yates correction, included in the statistical programme, was used in all calculations.

\section{Results}

Thirty-six sera, from dogs not vaccinated with wholecell bacterin, were analysed separately in a polyvalent ELISA with whole cells or recombinant antigens of $B$. burgdorferi. All 36 sera were positive by polyvalent ELISA with whole cells; antibody titres ranged from 320 to 40960 . Although there was less frequent reactivity of sera in assays with recombinant antigens (Table 1), antibodies to two or more highly specific recombinant antigens were detected in 32 sera $(89 \%)$. Two other test sera had antibodies to one recombinant antigen; the remaining two sera were negative in all assays with recombinant antigens.

Seropositivity rates for dogs not vaccinated with wholecell bacterin were highly variable and ranged from $8 \%$ for OspB and OspC to $89 \%$ for OspF antigens in ELISAs. Differences in these values were statistically

Table 1. Results of sero-analyses for total antibodies to sonicated whole-cell or purified recombinant antigens of $B$. burgdorferi in a polyvalent ELISA for 36 dogs with diagnosed borreliosis and no records of receiving $B$. burgdorferi vaccine

\begin{tabular}{|c|c|c|c|}
\hline \multirow{3}{*}{$\begin{array}{l}\text { Antigens } \\
\text { tested }\end{array}$} & \multicolumn{3}{|c|}{ ELISA results } \\
\hline & \multirow{2}{*}{$\begin{array}{l}\text { Number }(\%) \\
\text { seropositive* }^{*}\end{array}$} & \multicolumn{2}{|c|}{ Antibody titres } \\
\hline & & Geometric mean* & Range \\
\hline Whole cell & $36(100)$ & 6704 & $320-40960$ \\
\hline OspA & $4(11)$ & 538 & $160-10240$ \\
\hline OspB & $3(8)$ & 254 & $160-320$ \\
\hline OspC & $3(8)$ & 254 & $160-320$ \\
\hline OspE & $9(25)$ & 403 & $160-1280$ \\
\hline OspF & $32(89)$ & 3700 & $320-40960$ \\
\hline $\mathrm{p} 22$ & $9(25)$ & 373 & $160-2560$ \\
\hline p35 & $28(78)$ & 1999 & $160-40960$ \\
\hline p37 & $21(58)$ & 1966 & $160-40960$ \\
\hline p39 & $30(83)$ & 825 & $320-2560$ \\
\hline p41-G & $27(75)$ & 1532 & $160-5120$ \\
\hline
\end{tabular}

${ }^{*}$ In re-analyses of sera, numbers of dog sera positive to whole cells, OspA, OspB, OspC, OspE, OspF and p41-G antigens were the same as reported earlier [12] and are listed for comparison. Geometric means are for positive results only. 
significant $(\mathrm{z}=6.640, \mathrm{p}<0.001)$. In general, proportions of positive sera (ie., assay sensitivities) were highest in ELISAs that contained the following recombinant antigens in separate analyses: OspF, p35, p37, p39 and p41-G. Less frequent reactivity was noted when OspA, OspB, OspC, OspE and p22 antigens were included separately in ELISAs. Geometric mean titres were elevated when sera were screened in ELISAs with whole-cell, OspF, p35, p37, p39 and p41-G antigens. Maximal antibody titres ( $\geqslant 1$ in 10240$)$ were recorded when dog sera were tested with whole cells, OspA (one sample), OspF, p35 and p37 antigens.

Sixteen sera from dogs not vaccinated with whole-cell bacterin and positive by a polyvalent ELISA with whole-cell antigens were tested by immunoblotting methods to identify key banding patterns and check ELISA results. All 16 sera were considered positive in blots, based on reactivity to two or more highly specific, immunodominant proteins included in the panel of antigens. Although immune responses varied (Fig. 1), several proteins were frequently recognised immunologically. The most important were $\mathrm{p} 22$, OspE, OspF, p35, p37, p39, flagellin and p93. There were bands present for all of these antigens in eight dog sera including the positive control (Fig. 1, lanes 1, 9, 11, 12, $15,17,18,21)$. Other key, distinct bands, less frequently observed, corresponded to proteins with molecular masses of c. $31 \mathrm{kDa}(\mathrm{OspA}), 34 \mathrm{kDa}(\mathrm{OspB})$ and $23 \mathrm{kDa}(\mathrm{OspC})$. Four dog sera, negative by ELISA, were negative in blots.
For the same subset of 16 dog sera analysed by immunoblotting methods, ELISA results were examined to assess sensitivities for particular antigens (Table 2). In general, seropositivity rates were higher by immunoblotting than by ELISAs. Exceptions were noted for ELISAs with OspF and p35 antigens. However, differences in rates for OspC in an ELISA (13\% positive) and immunoblot analysis ( $56 \%$ positive) were statistically insignificant $(\mathrm{z}=1.070, \mathrm{p}=0.285)$.

Table 2. Comparative results for ELISA and immunoblotting when recombinant or whole-cell B. burgdorferi antigens were used to test sera from 16 dogs with diagnosed borreliosis and no records of receiving $B$. burgdorferi vaccine

\begin{tabular}{lcc}
\hline & \multicolumn{2}{c}{ Number (\%) seropositive by } \\
\cline { 2 - 3 } Antigen & ELISA & immunoblotting \\
\hline OspA & $1(6)$ & $1(6)$ \\
OspB & $3(19)$ & $5(31)$ \\
OspC & $2(13)$ & $9(56)$ \\
OspE & $4(25)$ & $10(63)$ \\
OspF & $15(94)$ & $11(69)$ \\
p22 & $5(31)$ & $11(69)$ \\
p35 & $13(81)$ & $13(81)$ \\
p37 & $11(69)$ & $13(81)$ \\
p39 & $15(94)$ & $16(100)$ \\
p41-G or flagellin & $13(81)$ & $15(94)$ \\
\hline
\end{tabular}

*All 16 sera were positive by ELISA and immunoblotting with whole-cell antigens. Recombinant antigens were also tested by ELISA, and reactivity was compared to banding patterns observed in immunoblot analysis with whole-cell antigen.

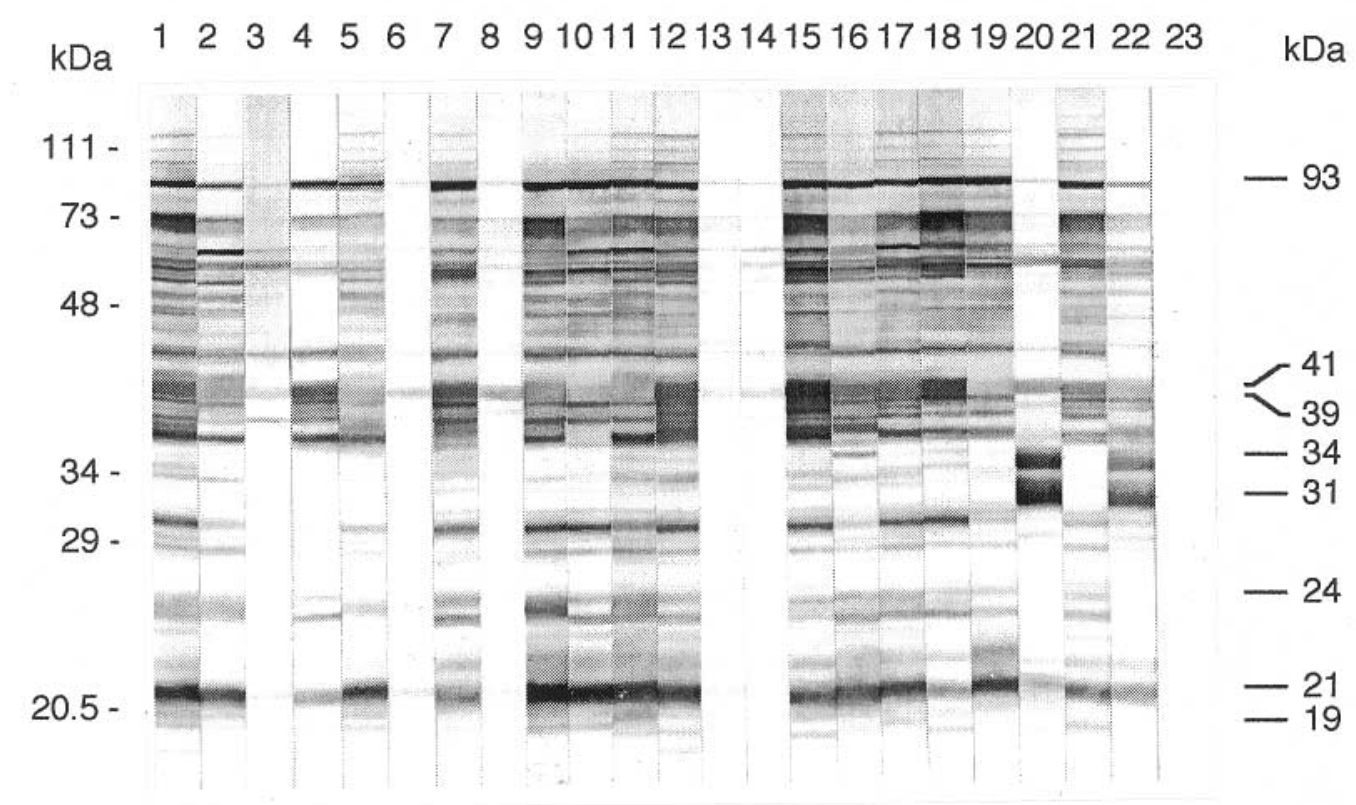

Fig. 1. Immunoblots of serum samples from dogs (lanes 1-21) and human subjects (lanes 22 and 23) with lysates of the B 297 strain of B. burgdorferi. Molecular masses (kDa) are marked in the left margin and key bands are indicated in the right margin. Lanes $\mathbf{1}-\mathbf{5}, \mathbf{7}, \mathbf{9 - 1 2}$ and 15-20 show reactivity patterns of seropositive dogs that had limb or joint disease; 6, 8, 13 and 14, (negative controls) indicate weak banding patterns; 21, dog serum of a positive control; 22, reactivity of a positive human control; 23, negative human control - no reactivity. All serum samples were from subjects not vaccinated against $B$. burgdorferi. 
Based on results of both assays, antibody reactivities to the following antigens were most frequent: OspF, p35, p37, p39 and flagellin (or p41-G). Antibody reactivities to $\mathrm{p} 22$, OspA, OspB, OspC and OspE in immunoblots were also noteworthy, but seropositivity rates for these antigens in ELISAs were relatively lower.

Polyvalent ELISA was used to detect antibodies in five dogs vaccinated with whole-cell bacterin. There were antibodies to three or more recombinant antigens when sera were tested separately. Following immunisations, all five $\operatorname{dogs}$ produced antibodies to OspA, OspB and p22 antigens; maximal antibody titres of 2560-20 480 were noted for reactions to OspA and OspB. Three dogs also had antibodies to p41-G or p37. All sera obtained before vaccinations gave negative results.

Minor cross-reactivity was noted in specificity tests. There was a weak reaction of a 1 in 160 dilution of a dog serum (with E. equi antibodies) to the p37 antigen, but there were no other false positive reactions of $E$. equi or E. canis antibodies in the dog or horse control sera to whole-cell or recombinant antigens of $B$. burgdorferi. Rabbit antiserum to $T$. pallidum reacted with whole-cell $B$. burgdorferi at a titre of 1280 , but was negative with all recombinant antigens. Rabbit serum with antibodies to B. burgdorferi was positive in ELISAs with whole cells, p35, p39 and p41-G antigens at titres ranging from 640 to 10240 . Similarly, rabbit antiserum to $L$. interrogans serovar icterohaemorrhagiae likewise reacted in an ELISA with whole-cell $B$. burgdorferi (titre 1280), but was negative in tests with recombinant antigens. Further, antiserum to $L$. interrogans serovar canicola and one dog serum with antibodies to $R$. rickettsii were negative when screened with whole-cell or recombinant antigens of $B$. burgdorferi. A second $\operatorname{dog}$ serum with $R$. rickettsii antibodies reacted weakly (titre 160) in an ELISA with the p35 antigen. The 12 negative control sera from Wyoming and checks for glutathione fusion products, PBSS, enzyme and substrate were negative in all assays.

\section{Discussion}

Purified recombinant antigens of B. burgdorferi can be used in ELISAs to help verify exposure of dogs to this agent and to aid in epidemiological studies. Based on results of ELISAs and immunoblotting, the most frequently recognised antigens indicating natural exposure to B. burgdorferi were OspF, p35, p37 and p39. These highly specific antigens also were found to be important markers for human [16, 18-20, 27-29] and equine [14] borreliosis. We suggest that reactivities to two or more of these antigens in separate ELISAs (particularly at titres $\geqslant 320$ ) or in blots indicate exposure of dogs to $B$. burgdorferi. Minor crossreactivity was noted for an ELISA with p35 antigen, but ELISAs with this or other recombinant antigens are more specific than those containing whole-cell $B$. burgdorferi antigen. Although antibodies to OspA, OspB, OspC, OspE and p22 antigens were detected less frequently in ELISAs, these reactions in conjunction with those noted for other key immunodominant peptides also provide supportive evidence of natural exposure to $B$. burgdorferi in dogs not vaccinated with whole-cell bacterin. However, the presence of antibodies does not necessarily indicate active infection.

Immune responses to $B$. burgdorferi vary in dogs $[9,30]$. Differences in antibody production among hosts could be due to severity of disease, differential expression of proteins by the pathogen in hosts, or duration of infection (ie., acute versus chronic infection). Dogs with signs of canine borreliosis have more extensive antibody reactivity to $B$. burgdorferi than infected dogs lacking signs of this disease [30]. Moreover, some proteins - such as OspC - are differentially expressed. For example, OspC is frequently present in $B$. burgdorferi in human infection with this spirochaete; IgM and $\operatorname{IgG}$ antibodies are produced to OspC during early and late Lyme borreliosis [18, 19]. Accordingly, antibody response to this peptide is considered to be an important indicator of $B$. burgdorferi infection in man. However, in the present study, the ELISA and immunoblotting results for dog sera indicated less frequent antibody reactivity to OspC. As in horses [31], this antigen appears to be of limited diagnostic importance. Therefore, determination of seropositivity should be based on an overall pattern of serum antibody reactivities to a panel of highly specific antigens.

The automated and easily standardised ELISAs, with the most important recombinant antigens (OspF, p35 and p39) and having similar assay sensitivity values, are useful for initial testing of dog sera in North America. Studies are needed to determine if these antigens are important markers for canine borreliosis in Europe where diverse strains of Borrelia in different genospecies occur. The reagents used in the present study should be tested separately until efficacy information is provided on the sensitivity and specificity of an ELISA with mixtures of recombinant antigens. Mixed antigens might improve assay sensitivity. However, the use of combined fusion proteins with glutathione as antigen in an ELISA might also decrease sensitivity if there is interference (ie., blocking) when antibody-antigen complex is being formed in plate wells. To characterise immune responses in dogs more precisely, further work is needed to develop class-specific ELISAs with p35 and p39 antigens to detect IgM antibody, which normally indicates recent infection, and IgG antibodies. A recombinant p93 antigen should be produced and included in future evaluations.

In general, immunoblotting results paralleled ELISA findings. In some cases, the former had greater 
sensitivities, although statistically insignificant, when seropositivity rates for $\mathrm{OspC}$, OspE and p22 were compared. These findings could be due to lower serum dilutions used in immunoblot analysis, other technical factors, such as the enzyme and substrate systems used, or to differences in binding of antibodies to antigen epitopes. However, we agree with others [13] that Western immunoblotting is a suitable standard for analyses of dog sera and is an acceptable alternative to a clinical definition of borreliosis. Culturing spirochaetes from mammalian tissues is the best evidence for active infection. Detection of B. burgdorferi DNA in blood samples is also helpful. However, culturing is expensive and often produces a low yield, and PCR methods for DNA detection also can be limited because of contamination or the effect of inhibitors, such as haemoglobin. Therefore, it is advised that, whenever possible, the ELISA be applied as an adjunct method along with the widely used immunoblotting procedures for routine analyses. If banding patterns for proteins of similar molecular mass (ie., p22 and OspC; p35 and p37) are difficult to interpret when lysed whole-cell antigen is used, then key recombinant antigens of $B$. burgdorferi can be used instead of whole cells in immunoblot analysis to identify more specific antibody reactivity to selected antigens [14].

The ELISA methods used in the present study were designed to assess possible natural $B$. burgdorferi infections in dogs not vaccinated with whole cells of this agent. Multiple bands were usually present in blots of sera from naturally infected dogs also positive by ELISAs. There was frequent reactivity to the p39 antigen in natural infections; this agrees with recent work [30]. However, other investigators [8, 9] reported that dogs vaccinated with $B$. burgdorferi bacterin can produce antibodies to this and several other antigens, such as OspA and OspB. The testing of sera from dogs vaccinated with $B$. burgdorferi bacterin in the present study verified the presence of antibodies to OspA, OspB, p22, p41-G and p37. Therefore, when testing dog sera in surveillance studies, it is imperative to determine from clinical records whether or not wholecell B. burgdorferi vaccines have been used. In surveillance programmes of dogs not vaccinated with $B$. burgdorferi bacterin, antibody responses to key immunodominant proteins of B. burgdorferi (particularly OspF, p35 and p39) in ELISAs or blots should receive special consideration when defining the geographic range of Lyme borreliosis and assessing regional risk for infection.

We thank Tia Blevins, Manchuan Chen and Hong Tao for technical assistance. This work was supported in part by federal Hatch funds administered by the US Department of Agriculture and grants from the Centers for Disease Control and Prevention (CCU-106581), the National Institutes of Health (PO-1-AI-30548 and AI-49988), the Mathers Foundation, the Arthritis Foundation, the American Heart Association and the State of Connecticut (Charles Goodyear Award). J.W.I. is supported by a fellowship from the L.P. Markey Charitable Trust and E.F. is a recipient of a clinical scientist award in translational research from the Burroughs Wellcome Fund.

\section{References}

1. Straubinger RK, Straubinger AF, Härter L et al. Borrelia burgdorferi migrates into joint capsules and causes an upregulation of interleukin-8 in synovial membranes of dogs experimentally infected with ticks. Infect Immun 1997; 65: $1273-1285$.

2. Kornblatt AN, Urban PH, Steere AC. Arthritis caused by Borrelia burgdorferi in dogs. J Am Vet Med Assoc 1985; 186: 960-964.

3. Levy SA, Magnarelli LA. Relationship between development of antibodies to Borrelia burgdorferi in dogs and subsequent development of limb/joint borreliosis. J Am Vet Med Assoc 1992; 200: 344-347.

4. Magnarelli LA, Anderson JF, Schreier AB, Ficke CM. Clinical and serologic studies of canine borreliosis. J Am Vet Med Assoc 1987; 191: 1089-1094.

5. Azuma Y, Kawamura K, Isogai H, Isogai E. Neurologic abnormalities in two dogs suspected (sic) Lyme disease. Microbiol Immunol 1993; 37: 325-329.

6. Grauer GF, Burgess EC, Cooley AJ, Hagee JH. Renal lesions associated with Borrelia burgdorferi infection in a dog. $\mathrm{J} \mathrm{Am}$ Vet Med Assoc 1988; 193: 237-239.

7. Levy SA, Duray PH. Complete heart block in a dog seropositive for Borrelia burgdorferi. Similarity to human Lyme carditis. J Vet Intern Med 1988; 2: 138-144.

8. Greene RT, Walker RL, Burgess EC, Levine JF. Heterogeneity in immunoblot patterns obtained by using four strains of Borrelia burgdorferi and sera from naturally exposed dogs. J Clin Microbiol 1988; 26: 2287-2291.

9. Guerra MT, Walker ED, Kitron U. Quantitative approach for the serodiagnosis of canine Lyme disease by the immunoblot procedure. J Clin Microbiol 2000; 38: 2628-2632.

10. Magnarelli LA, Anderson JF, Kaufmann AF, Lieberman LL, Whitney GD. Borreliosis in dogs from southern Connecticut. J Am Vet Med Assoc 1985; 186: 955-959.

11. Magnarelli LA, Anderson JF, Schreier AB. Persistence of antibodies to Borrelia burgdorferi in dogs of New York and Connecticut. J Am Vet Med Assoc 1990; 196: 1064-1068.

12. Magnarelli LA, Flavell RA, Padula SJ, Anderson JF, Fikrig E. Serologic diagnosis of canine and equine borreliosis: use of recombinant antigens in enzyme-linked immunosorbent assays. $J$ Clin Microbiol 1997; 35: 169-173.

13. Lindenmayer J, Weber M, Bryant J, Marquez E, Onderdonk A. Comparison of indirect immunofluorescent-antibody assay, enzyme-linked immunosorbent assay, and Western immunoblot for the diagnosis of Lyme disease in dogs. J Clin Microbiol 1990; 28: 92-96.

14. Fikrig E, Magnarelli LA, Chen M, Anderson JF, Flavell RA. Serologic analysis of dogs, horses, and cottontail rabbits for antibodies to an antigenic flagellar epitope of Borrelia burgdorferi. J Clin Microbiol 1993; 31: 2451-2455.

15. Lam TT, Nguyen T-PK, Fikrig E, Flavell RA. A chromosomal Borrelia burgdorferi gene encodes a 22-kilodalton lipoprotein, p22, that is serologically recognized in Lyme disease. J Clin Microbiol 1994; 32: 876-883.

16. Akin E, McHugh GL, Flavell RA, Fikrig E, Steere AC. The immunoglobulin (IgG) antibody response to OspA and OspB correlates with severe and prolonged Lyme arthritis and the IgG response to P35 correlates with mild and brief arthritis. Infect Immun 1999; 67:173-181.

17. Fikrig E, Barthold SW, Sun W, Feng W, Telford SR, Flavell RA. Borrelia burgdorferi P35 and P37 proteins expressed in vivo, elicit protective immunity. Immunity 1997; 6: 531-539.

18. Gerber MA, Shapiro ED, Bell GL, Sampieri A, Padula SJ. Recombinant outer surface protein C ELISA for the diagnosis of early Lyme disease. J Infect Dis 1995; 171: 724-727.

19. Padula SJ, Dias F, Sampieri A, Craven RB, Ryan RW. Use of recombinant OspC from Borrelia burgdorferi for serodiagnosis of early Lyme disease. J Clin Microbiol 1994; 32: 1733-1738.

20. Simpson WJ, Cieplak W, Schrumpf ME, Barbour AG, Schwan TG. Nucleotide sequence and analysis of the gene in Borrelia burgdorferi encoding the immunogenic P39 antigen. FEMS Microbiol Lett 1994; 119: 381-388.

21. Magnarelli LA, Anderson JF, McAninch JB. Serologic analyses of cottontail rabbits for antibodies to Borrelia burgdorferi. $J$ Clin Microbiol 1990; 28: 890-893.

22. Magnarelli LA, Anderson JF, Philip RN, Burgdorfer W. Antibodies to spotted fever-group rickettsiae in dogs and 
prevalence of infected ticks in southern Connecticut. Am J Vet Res 1982; 43: 656-659.

23. Postic D, Ras NM, Lane RS, Hendson M, Baranton G. Expanded diversity among California Borrelia isolates and description of Borrelia bissettii sp. nov. (formerly Borrelia group DN127). J Clin Microbiol 1998; 36: 3497-3504.

24. Dressler F, Ackermann R, Steere AC. Antibody responses to the three genomic groups of Borrelia burgdorferi in European Lyme borreliosis. J Infect Dis 1994; 169: 313-318.

25. Wilske B, Preac-Mursic V, Schierz G, Kühbeck R, Barbour AG, Kramer M. Antigenic variability of Borrelia burgdorferi. In Benach JL, Bosler EM (eds) Lyme disease and related disorders. (Annals of the New York Academy of Sciences, vol 539.) New York, New York Academy of Sciences. 1988: $126-143$.

26. Magnarelli LA, Anderson JF, Johnson RC, Nadelman RB, Wormser GP. Comparison of different strains of Borrelia burgdorferi sensu lato used as antigens in enzyme-linked immunosorbent assays. J Clin Microbiol 1994; 32: 1154-1158.

27. Fawcett PT, Rose C, Gibney KM, Chase CA, Kiehl B, Doughty
RA. Detection of antibodies to the recombinant P39 protein of Borrelia burgdorferi using enzyme immunoassay and immunoblotting. J Rheumatol 1993; 20: 734-738.

28. Gilmore RD, Kappel KJ, Johnson BJB. Molecular characterization of a 35-kilodalton protein of Borrelia burgdorferi, an antigen of diagnostic importance in early Lyme disease. $J$ Clin Microbiol 1997; 35: 86-91.

29. Gilmore RD, Murphree RL, James AM, Sullivan SA, Johnson BJB. The Borrelia burgdorferi 37-kilodalton immunoblot band (P37) used in serodiagnosis of early Lyme disease is the fla $\mathrm{A}$ gene product. J Clin Microbiol 1999; 37: 548-552.

30. Hovius JWR, Hovius KE, Oei A, Houwers DJ, van Dam AP. Antibodies against specific proteins of and immobilizing activity against three strains of Borrelia burgdorferi sensu lato can be found in symptomatic but not in infected asymptomatic dogs. J Clin Microbiol 2000; 38: 2611-2621.

31. Magnarelli LA, IJdo JW, Van Andel AE, Wu C, Padula SJ, Fikrig E. Serologic confirmation of Ehrlichia equi and Borrelia burgdorferi infections in horses from northeastern United States. J Am Vet Med Assoc 2000; 217: 1045-1050. 